then $D_{n}$ is also congruent to a diagonal matrix of the stated form. From the law of inertia ([1], p. 296-298) $D_{n}$ also has $p$ positive and $q$ negative elements and the proof is complete.

From this result Theorem 1 follows as a corollary since, if $w(x)$ is nonnegative, $C_{n}$ is positive definite and therefore congruent to a diagonal matrix with $n$ positive elements.

As a simple example consider a 2-point quadrature formula of the form

$$
\int_{-1}^{1}(3-5|x|) f(x) d x \simeq A_{1} f\left(x_{1}\right)+A_{2} f\left(x_{2}\right) .
$$

For this weight function the monomial integrals are $c_{0}=1, c_{1}=0, c_{2}=-1 / 2$, $c_{3}=0$. There are no real values of $x_{1}, x_{2}$ for which (3) can be made exact for $f(x)=1, x, x^{2}, x^{3}$. There are, however, an infinity of such formulas with real $x_{1}$, $x_{2}$ which are exact for $f(x)=1, x, x^{2}$ and Theorem 2 still applies. One such formula is

$$
\begin{aligned}
x_{1} & =\frac{1}{2} & x_{2} & =1 \\
A_{1} & =2 & A_{2} & =-1 .
\end{aligned}
$$

Computation Center

University of Kansas

Lawrence, Kansas

1. F. R. Gantmacher, Matrix Theory, Vol. 1, Chelsea, New York, 1959.

2. V. I. KRYLov, Approximate Calculation of Integrals, Macmillan, New York, 1962. (Translated from Russian).

3. G. Szego, Orthogonal Polynomials, Amer. Math. Soc. Colloquium Publ., v. 23, 1959.

\title{
A Partition Problem
}

\section{By M. H. McAndrew}

1. Introduction. The following theorem is proved: Given integers $a, b, c, d$, each $\geqq 2$, then either there exist integers $m, n$ with $|m-n| \leqq 1$, a partition of a into $m$ parts of which each part is coprime to $b$, and a partition of $c$ into $n$ parts, each part coprime to $d$; or the same conclusion holds with the roles of $a$ and $b$ reversed and the roles of $c$ and $d$ reversed.

This question arises in the investigation of the minimum length of input strings required to distinguish two partial automata. Elgot and Rutledge [1] deduce an upper bound for the length of such strings and by using the theorem quoted above show that this upper bound can be attained. In Section 4 we demonstrate by an example that the restriction " $a, b, c, d \geqq 2$ " cannot be relaxed.

2. Preliminary Lemmas. In the sequel, all variables are to be taken as strictly positive integers.

Lemma 1. If $l>1, l=\prod_{i=1}^{r} P_{i}$ where the $p_{i}$ are distinct primes, and if $m$ is even, then there is an a such that

$$
\begin{aligned}
(a, l) & =1, \\
(m-a, l) & =1 .
\end{aligned}
$$

Received November 16, 1962. 
Proof. For each $i$ the congruences

$$
\left\{\begin{array}{l}
a_{i} \not \equiv 0 \quad\left(\bmod p_{i}\right), \\
a_{i} \neq m\left(\bmod p_{i}\right)
\end{array}\right.
$$

have a common solution. For if $p_{i}=2$ then $a_{i}=1$ is a solution; otherwise, $p_{i} \geqq 3$ and equations (1) eliminate at most 2 of the $p_{i}$ congruence classes $\bmod p_{i}$.

Let $a \equiv a_{i}\left(\bmod p_{i}\right)$; by the Chinese Remainder Theorem such an $a$ exists (unique mod $l$ ). Clearly $a$ satisfies the conclusions of the lemma.

Lemma 2. If $p$ is a prime $\geqq 5$, then for any $a, m, l$, with $(l, p)=1$, there is $a b$ such that

$$
\begin{aligned}
& b \equiv a \quad(\bmod l) \\
& 1 \leqq b \leqq 3 l p / 5 \\
& (b, p)=(m-b, p)=1
\end{aligned}
$$

Proof. Let $x_{r}=a+r l$. At least $p-2$ of the integers $x_{1}, x_{2}, \cdots, x_{p}$ satisfy

and

$$
x_{r} \not \equiv 0 \quad(\bmod p)
$$

$$
x_{r} \not \equiv m \quad(\bmod p) \text {. }
$$

Let $y_{1}, y_{2}, \cdots, y_{p-2}$ be the least positive residues $(\bmod l p)$ of any $p-2$ of such solutions. Let $b=\operatorname{Min}\left(y_{i}\right)$. Then clearly equations (2), (4) are satisfied and

$$
\begin{aligned}
1 \leqq b & \leqq 3 l, \\
& \leqq 3 l p / 5, \quad \text { since } p \geqq 5 .
\end{aligned}
$$

Lemma 3. If $t=\prod_{i=1}^{s} p_{i}$ where the $p_{i}$ are distinct primes with $p_{s} \geqq 5$, and if $m$ is even, then there is a $b$ such that

$$
\begin{aligned}
(b, t) & =1, \\
(m-b, t) & =1,
\end{aligned}
$$

and

$$
1 \leqq b \leqq 3 t / \bar{\tau}
$$

Proof. If $s=1$, the result follows from Lemma 2 with $a=l=1$. If $s>1$, the result follows from Lemmas 1 and 2 with

$$
l=\prod_{i=1}^{s-1} p_{i}
$$

Definition. We define " $a$ is $P_{r}(b)$ " to mean "There exist $a_{1}, a_{2}, \cdots, a_{r}$ such that $a=\sum_{i=1}^{r} a_{i}$ and $\left(a_{i}, b\right)=1 \quad(i=1, \cdots, r)$."

Lemma 4 . If $m$ is an even integer $\geqq 6$ and $m \geqq 3 n / 5$ then $m$ is $P_{2}(n)$.

Proof. Let $n=\prod_{i=1}^{r} p_{i}^{\alpha_{i}}$ where the $p_{i}$ are distinct primes. Let $t=\prod_{i=1}^{r} p_{i}$ and suppose, without loss of generality, $p_{r}=\operatorname{Max}\left(p_{i}\right)$. We consider two cases.

(a) $P_{r} \geqq 5$. Let $b$ be defined as in Lemma 3 . Then 


$$
\begin{aligned}
1 \leqq b & \leqq 3 t / 5 \\
& \leqq 3 n / 5, \text { by definition of } t \\
& \leqq m, \quad \text { by hypothesis } \\
& <m, \quad \text { since }(m-b, n)=1 .
\end{aligned}
$$

Therefore, $c=m-b$ is a positive integer; i.e., since $m=b+c, m$ is $P_{2}(n)$.

(b) $P_{r}<5$. Now $t=1,2,3$ or 6 . It may be verified that at least one of the partitions $m=1+(m-1)$ or $m=5+(m-5)$ must have both parts prime to $t$. The result now follows.

Lemma 5 . If $m \geqq 2, n \geqq 1$ and

$$
\begin{array}{ll}
\text { either } & \text { (a) } m \geqq 3 n / 5+1 \text { and }(m, n) \not \equiv(5,6) \text {, } \\
\text { or } \quad \text { (b) } m \geqq(3 n+8) / 5, \\
\text { or } \quad \text { (c) } m \geqq n,
\end{array}
$$

then $m$ is either $P_{2}(n)$ or $P_{3}(n)$.

Proof. (a) If $m$ is an even integer $\geqq 6$, then $m$ is $P_{2}(n)$ by Lemma 4 . If $m$ is an odd integer $\geqq 7$, then $m-1$ is an even integer $\geqq 3 n / 5$ and hence $m-1$ is $P_{2}(n)$ by Lemma 4 ; i.e., $m$ is $P_{3}(n)$. The cases $m=2,3,4,5$ may be settled by inspection of the following partitions:

$$
\begin{aligned}
& 2=1+1, \\
& 3=1+1+1, \\
& 4=2+2=3-1 \quad(n \leqq 5 / 3(3)=5), \\
& 5=3+1+1=2+2+1 \quad(n \leqq 5 / 3(4)<7) .
\end{aligned}
$$

(b) If $(m, n) \not \equiv(5,6)$ the result follows from (a). If $(m, n)=(5,6)$ then $m<$ $(3 n+8) / 5$.

(c) If $n \geqq 3$ then $m \geqq n>3 n / 5+1$ and the result follows from (a). If $n=1$ or 2 , the result follows since $m=(m-1)+1=(m-2)+1+1$ and either $m-1$ or $m-2$ is odd.

Lemma 6 . If $u \geqq w \geqq 2$ and $u \geqq w+(3 v-2) / 5$ then $u$ is either $P_{u}(v)$ or $P_{w+1}(v)$.

Proof.

$$
u=\underbrace{1+1+\cdots 1}_{w-2}+(u-w+2)
$$

and $(u-w+2) \geqq(3 v+8) / 5$, by hypothesis. Hence, by Lemma $5(\mathrm{~b})$, $(u-w+2)$ is either $P_{2}(v)$ or $P_{3}(v)$. Therefore, $u$ is either $P_{w}(v)$ or $P_{w+1}(v)$.

\section{Main Theorem.}

Theorem 1. If $a, b, c, d$ satisfy

$$
a, b, c, d, \geqq 2
$$

then for some $m, n$ with $|m-n| \leqq 1$, either $a$ is $P_{m}(b)$ and $c$ is $P_{n}(d)$, or $b$ is $P_{m}(a)$ and $d$ is $P_{n}(c)$. 
Proof. Suppose the theorem is false. We shall deduce a contradiction. We consider two cases.

(i) Either $(a, b),(b, a),(c, d)$ or $(d, c)=(5,6)$. Suppose, without loss of generality, $(a, b)=(6,5)$. Now $6=4+2$; hence 6 is $P_{2}(5)$. Therefore $c$ is not $P_{2}(d)$ or $P_{3}(d)$. By the converse of Lemma $5(\mathrm{c}), d>c$; i.e.,

$$
d \geqq c+1 \text {. }
$$

Now $6=3+3=3+2+1=3+1+1+1=2+1+1+1+1=1+$ $1+1+1+1+1$; i.e., 6 is $P_{2}(5), P_{3}(5), P_{4}(5), P_{5}(5), P_{6}(5)$. Now $c$ is $P_{c}(d)$, trivially. Therefore

$$
c \geqq 8 \text {. }
$$

Finally 5 is $P_{5}(6)$, trivially; hence $d$ is not $P_{4}(c)$ or $P_{5}(c)$. Therefore, by the converse of Lemma 6 with $u=d, \quad v=c$, and $w=4$,

$$
d<(3 c+18) / \tilde{5}
$$

From (6) and (8),

$$
c+1<(3 c+18) / 5, \quad 2 c<13,
$$

which contradicts (7).

(ii) None of $(a, b),(b, a),(c, d),(d, c)=(5,6)$. Without loss of generality, suppose $a \geqq b$. Then by Lemma $5(\mathrm{c}) \quad a$ is either $P_{2}(b)$ or $P_{3}(b)$. Hence $c$ is neither $P_{2}(d)$ nor $P_{3}(d)$; by the converse of Lemma 5 ,

$$
\begin{aligned}
& c<d, \\
& c<3 d / 5+1 .
\end{aligned}
$$

Similarly from (9) we deduce

$$
\begin{aligned}
& b<a \\
& b<3 a / 5+1
\end{aligned}
$$

Suppose without loss of generality, $a \geqq d$. In (10)

$$
c<3 a / 5+1 \text {. }
$$

From (12) and (13)

$$
\begin{aligned}
3 b+5 c & <3(3 a / 5+1)+5(3 a / 5+1) \\
& <\frac{24}{5} a+8 \\
& <5 a+8 \\
& \leqq 5 a+7 .
\end{aligned}
$$

i.e. $a \geqq(c-1)+(3 b-2) / \tilde{5}$. Hence, by Lemma 6 , $a$ is either $P_{c-1}(b)$ or $P_{c}(b)$. Now $c$ is $P_{c}(d)$ trivially; hence we have the required contradiction.

4. Remark. The following theorem shows that Theorem 1 is best possible in that condition (5) cannot be relaxed.

Theorem 2. For arbitrary $K$ there exist $a, b, c, d$, with 


$$
\begin{gathered}
a=1, \\
b, c, d,>K
\end{gathered}
$$

and such that the conclusion of Theorem 1 is false.

Proof. Let $b=N, d=2^{\dot{M}}-2, c=2^{-r}(d !)$, where $r$ is chosen to make $c$ an odd integer. Clearly $a$ is $P_{s}(b)$ only for $s=1$. Now $c$ is not $P_{1}(d)$, provided $d>3$, and not $P_{2}(d)$ since an odd integer cannot be the sum of two odd integers. Hence, we cannot find partitions of $a, c$ satisfying the conclusions of Theorem 1.

Suppose $d$ is $P_{s}(c)$. Then

$$
d=d_{1}+d_{2}+\cdots+d_{s}
$$

where each $d_{i} \leqq d$ and $\left(d_{i}, c\right)=1$. Now $c$ is divisible by all odd integers $<d$; therefore $d_{i}$ is a power of 2 . I.e.,

$$
d=2^{r_{1}}+2^{r_{2}}+\cdots 2^{r_{s}} .
$$

Since $d=2^{M}-2$ there are at least $M-1$ summands in (14). I.e., if $d=P_{s}(c)$, then $s \geqq M-1$. But clearly if $b$ is $P_{s}(a)$, then $s \leqq N$. If we now choose $M-1>$ $N+1$ and $M, N$ large enough to ensure $b, c, d>K$, the conclusion of Theorem 2 follows.

International Business Machines Corporation

Thomas J. Watson Research Center

Yorktown Heights, New York

1. C. C. Elgot \& J. D. Rutledge, "Operations on Finite Automata," Proceedings of the Second Symposium on Switching Circuit Theory and Logical Design (AIEE: 5-134), October, 1961.

\section{Approximations to Kelvin Functions}

\section{By F. D. Burgoyne}

While preparing a digital computer program to examine the behavior of largetaper hub flanges, it was found necessary to use approximations to the Kelvin functions ber $x$, bei $x$, $\operatorname{ker} x$, and kei $x$, and to their first derivatives. To obtain full machine accuracy, the approximations were required to be correct to nine significant figures. Several tabulations of these functions exist, but the only ones considered to be sufficiently accurate were those of Lowell [1] and Nosova [2]; however, limitations of internal memory in the computer used precluded the possibility of storing such tables and interpolating.

The functions actually required were $Z_{i}(x)$ and $Z_{i}^{\prime}(x)(1 \leqq i \leqq 4)$, where

$$
\begin{aligned}
& Z_{1}(x)=\text { ber } x \\
& Z_{2}(x)=- \text { bei } x \\
& Z_{3}(x)=-\frac{2}{\pi} \operatorname{kei} x \\
& Z_{4}(x)=-\frac{2}{\pi} \operatorname{ker} x ;
\end{aligned}
$$

Received Feburary 5, 1962. 